УДК ОО $4+658$

$10.17213 / 2075-2067-2020-5-224-240$

\title{
ПРОГНОЗИРОВАНИЕ ДИНАМИКИ ИНДИКАТОРОВ УРОВНЯ РАЗВИТИЯ ЭКОНОМИКИ СТРАНЫ: МОДЕЛИ, МЕТОДЫ, ИНСТРУМЕНТАЛЬНЫЕ СРЕДСТВА (НА ПРИМЕРЕ ГЕРМАНИИ, РОССИИ И ШВЕЦИИ ${ }^{1}$
}

\author{
(C) 2020 г. $\quad$ Г. Н. Хубаев \\ Ростовский государственный экономический университет (РИНХ), \\ 2. Ростов-на-Дону, Россия
}

Целью исследования является анализ и прогнозирование динамики индикаторов уровня развития экономики страны. Расчеты проведены на данных Всемирного банка по Германии, России и Швеции.

Методологическую базу исследования представляют экономическая теория, идеи и рекомендации, содержащиеся в работах Мориса Алле (M. Allais) и Фредерика Хайека (F. Hayek), экономико-математические методы и модели.

Результаты исследования. Выделено с использованием оригинальных методов подмножество определяющих факторов из исходного множества большой мощности. Построено более двух десятков регрессионных моделей для прогнозирования динамики ВВП странь и ожидаемой продолжительности жизни граждан $-R^{2}$ скорр $\approx 0,9 ; F$ кр $>>100$, $b_{i} / \sigma_{b i}>>2$. Средняя ошибка прогноза за 10-летний период по исследуемым странам - менее $5 \%$ при лаге независимых переменных от одного до трех лет. Проведен расчет ошибок функиий отклика с использованием имитационного моделирования. Обнаружено, что величина ошибок функций отклика даже у моделей с близкими численными значениями статистических характеристик качества существенно отличается.

Перспектива исследования состоит в том, что полученное в прочессе исследований доказательство статистической и содержательной обоснованности влияния на динамику индикаторов уровня развития экономики выделенного оригинального подмножества определяющих факторов обеспечило возможность исполнительной государственной власти в различных странах мира более целенаправленно и активно содействовать ускоренному развитию экономики, ориентируясь на созданные конкретные модели и конкретные независимые переменные.

Ключевые слова: динамика индикаторов; ВВП страны; продолжительность жизни; регрессионные модели; имитационное моделирование; ошибки прогноза.

\section{FORECASTING THE DYNAMICS OF INDICATORS OF THE LEVEL OF DEVELOPMENT OF THE COUNTRY'S ECONOMY: MODELS, METHODS, TOOLS (ON THE EXAMPLE OF GERMANY, RUSSIA AND SWEDEN)}

1 Статья подготовлена по результатам исследований, выполненных при поддержке Российского фонда фундаментальных исследований (РФФИ) — проект 18-010-00806/18 «Уровень жизни населения административнотерриториальных образований: выявление, исследование, анализ и оценка значимости определяющих факторов (для последующей оптимизации в условиях ограниченных ресурсов)». 


\section{(C) 2020 G. N. Khubaev}

\section{Rostov State University of Economics (RSUE), Rostov-on-Don, Russia}

The purpose of the study is to analyze and forecast the dynamics of indicators of the level of development of the country's economy. The calculations are based on world Bank data for Germany, Russia and Sweden.

The methodological basis of the research is economic theory, ideas and recommendations contained in the works of Maurice Allais and Frederick Hayek, economic and mathematical methods and models.

Research result. Using original methods, a subset of determining factors from the original high-power set is selected. More than two dozen regression models were built to predict the dynamics of the country's GDP and the life expectancy of citizens $-R^{2}$ скорр $\approx 0,9 ; F \mathrm{\kappa p}>>100$, $b_{i} / \sigma_{b i}>>2$. The average forecast error over a 10-year period for the studied countries is less than 5\% with a lag of independent variables from one to three years. Errors of response functions are calculated using simulation modeling. It is found that the magnitude of errors in response functions differs significantly even for models with similar numerical values of statistical quality characteristics.

The prospect study is that the research evidence of the statistical and substantive validity of the influence on the dynamics of indicators of level of economic development highlighted in the original subset of determinants provided the opportunity for the Executive state power in various countries of the world more deliberately and actively promote the accelerated development of the economy, focusing on a specific model and specific independent variables.

Key words: dynamics of indicators; country's GDP; life expectancy; regression models; simulation modeling; forecast errors.

Введение. В мире две сотни стран ориентируются на рыночную экономику, а резульmaты социально-экономического развития этих стран почему-то разные. Да и скорость появления позитивных изменений в экономике стран мира тоже разная (см. данные $\mathrm{OOH}$ и Всемирного банка). Причём, как оказалось, ни климат, ни площадь территории, ни географическая широта, ни даже наличие или отсутствие полезных ископаемых почему-то не оказывают существенного влияния на самые главные экономические показатели и результаты.

Странная ситуация, на первый взгляд, получается: все заинтересованы, но не все могут (или не знают, как изменить ситуацию, переломить исходное состояние в нужном направлении). Но ведь для того, чтобы успешно управлять процессом ускоренного развития экономики страны и ростом уровня жизни граждан, необходимо знать ответьл на вопросы:
- на какие факторы-показатели следует воздействовать, чтобы получить нужный результат — ожидаемое значение функции отклика;

- какова степень влияния этих факторов на уровень развития экономики и уровень жизни граждан;

- как сформировать оптимальный состав независимых переменных для прогнозирования функции отклика;

- и как определить, насколько заметно отражается на динамике функиии отклика скорость приращения некоторых содержательно и статистически значимых факторов;

- главное - с каким запаздыванием (лагом) проявляется влияние тех или иных факторов на динамику функции отклика и какова степень этого влияния;

- и, конечно, каковы затраты ресурсов на реализацию каждого фактора, на величину инвестищий в каждый фактор-показатель. 
Но, оказывается, совсем не просто сформировать необходимые исходные данные, чтобы ответить на поставленные вопросы. Ведь в открытых статистических данных, сформированных ООН и Всемирным банком, представлены сведения о значениях нескольких тысяч экономических показателей, характеризующих уровень социально-экономического развития стран мира. И как выбрать необходимое подмножество определяющих факторов из множества столь большой мощности?

Здесь мы, продолжая ранее начатые исследования (см., например, [1-3]), используем оригинальный метод выделения (с оценкой величины лага) ограниченного подмножества объектов (факторов, признаков, показателей) из исходного множества достаточно большой мощности, содержащего сотни и тысячи объектов. При выполнении расчетов использованы данные Всемирного банка, характеризующие уровень социального и экономического развития Германии,

\section{России и Швеции.}

[Пояснение. Почему выбраны именно эти три страны? Во-первых, Германия - это реальный «донор» ЕС, который со времен «немецкого чуда» (порожденного в начале 50-х годов прошлого века в результате действий команды Людвига Эрхарда) продолжает успешно выявлять и реализовывать приоритетные, как оказалось, направления развития страны. Россия - это страна, 20 лет назад приступившая к социально-экономическим преобразованиям в условиях массовой нищеты, убыли населения, развала экономики (см., например, $[4,11]$ и/или в [1] страницу «Как мы жили 20 лет назад»), смогла за ничтожно малый (по историческим меркам) промежуток времени осуществить (по результатам социально-экономического развития) поразительный скачок, позволивший добиться непрерывного (с 2003 г.) роста ожидаемой продолжительности жизни граждан (ОПЖ) и ежегодного увеличения в течение 8 последних лет количества лиц старше 100 лет. Швеция - это страна не только с относительно ограниченным запасом используемых полезных ископаемых, но и почему-то с долгоживущими (с 2003 г. ОПЖ $\geq 80$ лет) и к тому же с самыми счастливыми в мире гражданами (наряду с Финляндией, Норвегией и Данией), такое заключение вынесли эксперты британского центра Legatum, опубликовавшие «рейтинг счастья», в котором оценены 104 страны мира.

Во-вторых, мы полагаем, что органы исполнительной власти этих стран вместе с Всемирным банком осуществляют достаточно строгий контроль за уровнем достоверности данных государственной статистики.]

\section{1. Регрессионные модели для прогно-} зирования ОПЖ граждан и ВВП страны с оценкой характеристик потребительского качества. В процессе исследований, направленных на выделение определяющих факторов-показателей, осуществлялся предварительный отбор искомого подмножества факторов из множества претендентов с использованием метода $[8,13]$. В последующем уменьшение размерности пространства факторов выполнено путем экспертных опросов по методу пошагового уточнения ранжирования объектов-факторов [7]. В результате исходный состав факторов уменьшался в среднем на порядок. При этом стало возможным проводить сравнительный анализ статистических характеристик качества построенных регрессионных моделей и *разной структуры, и *с разным составом факторов - независимых переменных.

Оценка значимости факторов и величины лага влияния проведена при использовании исходной информации в относительных единицах (отношение численных значений показателей по годам рассматриваемого периода).

По каждой из стран представлены *сформированный состав независимых переменных в регрессионных моделях, *статистические характеристики степени влияния переменных на функцию отклика с указанием величины лага.

При выделении подмножеств определяющих факторов, оказывающих основное влияние на исследуемые функции отклика (на ВВП страны и ожидаемую продолжительность жизни граждан), использованы сведения из базы Всемирного банка за период в 10-12 лет по 2015 год включительно. Одновременно оказалось, что с декабря 2019 года и в 2020 году для оценки потребительского качества построенных моделей можно воспользоваться содержащимися в базе Всемирного банка данными по ВВП и ОПЖ анали- 
зируемых стран дополнительно ещё за 2-3 года, т.е. за 2016, 2017 и иногда за 2018 годы. Поэтому при сравнительном анализе прогнозных свойств построенных моделей использованы сведения о значениях функций отклика за период до 2017 года или, при наличии требуемой информации, до 2018 года, причем в расчетах использовались и фактические данные (абсолютная шкала), и относительные значения (ежегодный прирост значений функций отклика). Что касается независимых переменных, то они, как и ранее, представлены в относительных единицах за весь период до 2015 года - как приращения за период от одного до трех лет, т.е. с учетом запаздывания с влиянием на функцию отклика (лага).

Для каждой страны и рассматриваемых функций отклика представлены регрессионные модели, обладающие лучшими статистическими характеристиками потребительского качества и минимальными ошибками функций отклика (ВВП и ОПЖ).

[Пояснение 2. Характеристики потребительского качества построенных моделей оцениваются используемыми в статистике показателями: $\boldsymbol{b} / \boldsymbol{\sigma}_{\mathbf{b i}}, \boldsymbol{R}_{\mathbf{c k}}^{\mathbf{2}}, \boldsymbol{F} \mathbf{\kappa p . ~ В ~ п р е д с т а в - ~}$ ленных ниже моделях при неизменной функции отклика изменяется только состав независимых переменных.]
После выполнения расчетов по каждой из функций отклика в изучаемых странах получены регрессионные модели с достаточно хорошими и практически одинаковыми статистическими характеристиками потребительского качества как для абсолютных, так и относительных значений. Однако, исходя из прикладной полезности для управления инвестиционной деятельностью государства (путем изменения налоговой политики, компенсации доли кредита, иелевой финансовой помощи административно-территориальным образованиям и др.), для сравнительной оценки потребительского качества выделены и здесь представлены модели с лучшими статистическими характеристиками и функцией отклика в относительных единицах, т.е. с величиной ежегодного приращения функции отклика - приращения ВВП на душу населения, ППС; ВВП на одного работающего и ОПЖ граждан. Исходная информация представлена в таблицах 1-7.

1.1. Функция отклика $Y 1-$ Ожидаемая продолжительность жизни граждан за период с 2006 по 2017 годы.

*Швеция. Исходная информация представлена в таблице 1.

Все три модели имеют вид: $Y 01=b 1 * X 1$, где:

\section{Исходная информация по Швеции - ОПж}

Таблица 1

\begin{tabular}{|c|c|c|c|c|c|}
\hline Год & $\begin{array}{c}\text { Значения } \\
\text { ОПЖ, } Y_{i}\end{array}$ & $\begin{array}{c}\text { Значения } \\
Y_{i} Y_{i-1}\end{array}$ & $\begin{array}{c}\text { Модель 1. } \\
\text { Значения } X 1\end{array}$ & $\begin{array}{c}\text { Модель 2. } \\
\text { Значения } X 1\end{array}$ & $\begin{array}{c}\text { Модель 3. } \\
\text { Значения } X 1\end{array}$ \\
\hline 2006 & 80,75 & & 1,088659 & 1,012386 & 0,97913 \\
\hline 2007 & 80,9 & 1,001858 & 1,07342 & 1,023981 & 1,007906 \\
\hline 2008 & 81,1 & 1,002472 & 1,062381 & 1,014645 & 1,002307 \\
\hline 2009 & 81,35 & 1,003098 & 1,021045 & 1,022601 & 0,974342 \\
\hline 2010 & 81,45 & 1,001229 & 0,994739 & 0,983958 & 0,952197 \\
\hline 2011 & 81,80 & 1,004312 & 1,355728 & 1,303169 & 1,014874 \\
\hline 2012 & 81,70 & 0,998807 & 1,042476 & 0,999 & 1,049969 \\
\hline 2013 & 81,95 & 1,003075 & 1,034755 & 0,999828 & 1,020248 \\
\hline 2014 & 82,25 & 1,003631 & 1,022794 & 1,020674 & 0,99603 \\
\hline 2015 & 82,20 & 0,999407 & 1,016734 & 1,017482 & 0,994964 \\
\hline 2016 & 82,30 & 1,001246 & & & \\
\hline 2017 & 82,30 & 1,0 & & & \\
\hline
\end{tabular}


1) Модель с $X 1-X 1-$ относительное приращение внутренних государственных расходов на здравоохранение на душу населения, ППС (за один год, текущие, в долларах). Статистические характеристики качества модели:

$$
\begin{gathered}
b 1=0,93 ; \sigma_{\mathrm{b1}}=0,028 ; b 1 / \sigma_{\mathrm{b1}}=32,8 ; \\
R^{2}=0,99 ; R_{\text {ск }}^{2}=0,88 ; F \kappa p=1077,98 . \\
\text { Значимость } F \text { кр }=8,08 \mathrm{E}-10 .
\end{gathered}
$$

2) Модель с $X 1$ - относительное приращение внутренних государственных расходов на здравоохранение (за один год, в \% от государственных расходов). Статистические характеристики качества модели:

$$
\begin{gathered}
b 1=0,96 ; \sigma_{\mathrm{b1}}=0,027 ; b 1 / \sigma_{\mathrm{b} 1}=35,4 ; \\
R^{2}=0,99 ; R_{\text {ск }}^{2}=0,88 ; F_{\text {кр }}^{=} 1255,1 . \\
\text { Значимость } F \text { кр }=4,41 \mathrm{E}-10 .
\end{gathered}
$$

3) Модель с $X 1$ - относительное приращение оплаты труда работников (за два года, в \% от расходов). Статистические характеристики качества модели:

$$
\begin{gathered}
b 1=1,002 ; \sigma_{\mathrm{b} 1}=0,008 ; b 1 / \sigma_{\mathrm{b} 1}=118,96 ; \\
R^{2}=0,99 ; R_{\mathrm{ck}}^{2}=0,89 ; \boldsymbol{F} \mathbf{k p}=14151,4 . \\
\text { Значимость } F \text { кр }=\mathbf{2 , 7 9 E}-14 . \\
\text { Модели для прогнозирования ОПЖ }
\end{gathered}
$$
по Германии и России построены нами в [14].

1.2. Функция отклика $\boldsymbol{Y 2}$ - ВВП на душу населения, ППС (в постоянных международных долларах 2011 года).

*Германия. На конец 2019 года по Германии в базе Всемирного банка содержались сведения о ВВП на душу населения, ППС (в долларах 2011 г.) до 2018 года, а при выделении определяющих факторов использовались данные до 2015 года включительно. Поэтому качество построенных прогнозных моделей можно оценивать и по данным с 2016 по 2018 годы, и за весь период с 2009 по 2018 год. Функции отклика - это и абсолютные значения ВВП на душу населения, ППС, и данные о ежегодном приращении этого фактора, а для независимых переменных лаг составлял от одного до трех лет.

Исходная информация представлена в таблице 2.

1) Модель 1. X1 - относительное приращение валового накопления основного капитала (за три года, в \% от ВВП). Статистические характеристики качества модели:

$$
\begin{gathered}
b 1=1,0012 ; \sigma_{\mathrm{b} 1}=0,011 ; b 1 / \sigma_{\mathrm{b} 1}=90,4 ; \\
R^{2}=0,99 ; R_{\text {ск }}^{2}=0,89 ; F \kappa p=8172,6
\end{gathered}
$$

Значимость $F$ кр $=2,5 \mathrm{E}-13$.

2) Модель 2. X1 - относительное приращение экспорта промышленных товаров (за три года, в \% от объема экспорта товаров). Статистические характеристики качества модели:

$$
\begin{gathered}
b 1=1,014 ; \sigma_{\mathrm{b} 1}=0,014 ; b 1 / \sigma_{\mathrm{b} 1}=71,3 ; \\
R^{2}=0,99 ; R_{\text {ск }}^{2}=0,89 ; F \kappa \mathrm{Kp}=5086,4 \\
\text { Значимость } F \text { кр }=1,7 \mathrm{E}-12 .
\end{gathered}
$$

*Россия. Исходная информация представлена в таблице 3 .

1) Модель 1. X1 - приращение экспорта коммерческих услуг (за один год, в текущих ценах). Статистические характеристики качества модели:

$$
\begin{gathered}
b 1=0,906 ; \sigma_{\mathrm{b1}}=0,04 ; b 1 / 6_{\mathrm{b1}}=21,8 ; \\
R^{2}=0,97 ; R_{\text {ск }}^{2}=0,89 ; F \kappa p=475,6 . \\
\text { Значимость } F \text { кр }=9,19 \mathrm{E}-10 .
\end{gathered}
$$

2) Модель 2. X1 - приращение объема торговли услугами (за три года, в \% от ВВП); $X 2$ - приращение внутренних государственных расходов на здравоохранение на душу населения (за один год). Статистические характеристики качества модели: $b 1=0,53 ; b 2=0,45 ; \sigma_{b 1}=0,05 ; \sigma_{b 2}=0,045$;

$b 1 / \sigma_{\mathrm{b} 1}=10,21 ; b 2 / \sigma_{\mathrm{b} 2}=10,05 ; R^{2}=0,99$; $R_{\text {ск }}^{2}=0,9 ;$ Fкр $=1627,9$.

Значимость Fкр = 3,03E-12.

*Швеция. Исходная информация представлена в таблице 4.

1) Модель 1. X1 - приращение величины сборов за использование интеллектуальной собственности (за один год, в текущих ценах). Статистические характеристики качества модели:

$$
\begin{gathered}
b 1=0,88 ; \sigma_{\mathrm{b} 1}=0,04 ; b 1 / \sigma_{\mathrm{b} 1}=20,9 ; \\
R^{2}=0,98 ; R_{\text {ск }}^{2}=0,87 ; F \text { кр }=436,5 . \\
\text { Значимость } F \text { кр }=2,89 \mathrm{E}-08 .
\end{gathered}
$$

2) Модель 2. $X 1$ - приращение объема экспорта коммерческих услуг (за один год, в текущих ценах). Статистические характеристики качества модели:

$$
\begin{gathered}
b 1=0,93 ; \sigma_{\mathrm{b1}}=0,03 ; b 1 / 6_{\mathrm{b1}}=28,4 ; \\
R^{2}=0,99 ; R_{\text {ск }}^{2}=0,88 ; F \text { кр }=805,5 . \\
\text { Значимость } F \text { кр }=2,57 \mathrm{E}-09 .
\end{gathered}
$$

3) Модель 2. X1 - Услуги: приращение добавленной стоимости на одного работника (за три года). Статистические характеристики качества модели:

$$
\begin{gathered}
b 1=0,98 ; \sigma_{b 1}=0,013 ; b 1 / \sigma_{b 1}=72,08 \\
R^{2}=0,99 ; R_{\text {ск }}^{2}=0,89 ; F \kappa p=5195,5 \\
\text { Значимость } F \text { кр }=1,53 E-12
\end{gathered}
$$




\section{Исходная информация по Германии - ВВП на душу населения, ППС}

\begin{tabular}{|c|c|c|c|c|}
\hline Год & $\begin{array}{c}\text { Значения } Y_{i} \text { ВВП } \\
\text { нашу нушасения, ППС } \\
\text { (в долларах 2011 г.) }\end{array}$ & Значения $Y_{i} Y_{i-1}$ & $\begin{array}{c}\text { Модель 1. } \\
\text { Значения } X 1\end{array}$ & $\begin{array}{c}\text { Модель 2. } \\
\text { Значения } X 1\end{array}$ \\
\hline 2005 & 37703,93 & & & 1,016 \\
\hline 2006 & 39143,17 & 1,038 & 1,050 & 0,988 \\
\hline 2007 & 40473,53 & 1,034 & 1,066 & 0,954 \\
\hline 2008 & 40989,44 & 1,013 & 0,967 & 0,960 \\
\hline 2009 & 38784,45 & 0,946 & 0,966 & 0,996 \\
\hline 2010 & 40428,72 & 1,042 & 0,997 & 1,014 \\
\hline 2011 & 42692,52 & 1,056 & 1,050 & 1,025 \\
\hline 2012 & 42822,1 & 1,003 & 1,014 & 1,015 \\
\hline 2013 & 42914,48 & 1,002 & 0,987 & 1,005 \\
\hline 2014 & 43666,77 & 1,018 & 0,987 & 1,007 \\
\hline 2015 & 44043,18 & 1,009 & & \\
\hline 2016 & 44668,7 & 1,014 & & \\
\hline 2017 & 45462,03 & 1,018 & & \\
\hline 2018 & 45959,36 & 1,011 & & \\
\hline
\end{tabular}

Исходная информация по России - ВВП на душу населения, ППС

\begin{tabular}{|c|c|c|c|c|c|}
\hline \multirow{3}{*}{ Год } & \multirow{3}{*}{$\begin{array}{c}\text { Значения Y ВВП } \\
\text { на душу населения, ППС } \\
\text { (в долларах } 2011 \text { г.) }\end{array}$} & \multirow{3}{*}{$\begin{array}{l}\text { Значения } \\
Y Y_{i-1}\end{array}$} & \multicolumn{3}{|c|}{$\begin{array}{c}\text { Относительные значения } \\
\text { независимых переменных за 2004-2015 гг. }\end{array}$} \\
\hline & & & \multirow{2}{*}{$\begin{array}{c}\text { Модель } 1 \\
X 1\end{array}$} & \multicolumn{2}{|c|}{ Модель 2} \\
\hline & & & & $X 1$ & $X 2$ \\
\hline 2006 & 20970,2 & 1,09 & 1,25 & 0,88 & 1,28 \\
\hline 2007 & 22798,9 & 1,09 & 1,25 & 0,8 & 1,35 \\
\hline 2008 & 24006 & 1,05 & 1,24 & 0,77 & 1,31 \\
\hline 2009 & 22121,9 & 0,92 & 1,23 & 0,831 & 1,32 \\
\hline 2010 & 23325,9 & 1,05 & 1,29 & 0,89 & 1,33 \\
\hline 2011 & 24310 & 1,04 & 0,8 & 1,08 & 0,84 \\
\hline 2012 & 25156,4 & 1,03 & 1,07 & 1,02 & 1,08 \\
\hline 2013 & 25551 & 1,02 & 1,18 & 0,89 & 1,23 \\
\hline 2014 & 25284,6 & 0,99 & 1,07 & 0,87 & 1,13 \\
\hline 2015 & 24516,5 & 0,974 & 1,12 & 1,06 & 1,05 \\
\hline 2016 & 24416,6 & 0,99 & 0,94 & 1,24 & 0,91 \\
\hline 2017 & 24790,6 & 1,02 & 0,78 & 1,33 & 0,64 \\
\hline
\end{tabular}


Исходная информация по Швеции - ВВП на душу населения, ППС

Таблица 4

\begin{tabular}{|c|c|c|c|c|c|c|}
\hline Год & $\begin{array}{c}\text { Значения } Y_{i} \text { ВВП } \\
\text { на душу населения, } \\
\text { ПС (в долларах 2011 г.) }\end{array}$ & $\begin{array}{c}\text { Значения } \\
Y_{i} Y_{i-1}\end{array}$ & Год & $\begin{array}{c}\text { Модель 1. } \\
\text { Значения } X 1\end{array}$ & $\begin{array}{c}\text { Модель 2. } \\
\text { Значения } X 1\end{array}$ & $\begin{array}{c}\text { Модель 3. } \\
\text { Значения } X 1\end{array}$ \\
\hline 2006 & 42966,78 & 1,04 & 2006 & 1,12 & 1,14 & 1,07 \\
\hline 2007 & 44102,87 & 1,03 & 2007 & 1,09 & 1,24 & 1,03 \\
\hline 2008 & 43516,86 & 0,98 & 2008 & 1,11 & 1,11 & 1,01 \\
\hline 2009 & 40905,8 & 0,94 & 2009 & 0,89 & 0,84 & 0,98 \\
\hline 2010 & 42988,81 & 1,05 & 2010 & 0,81 & 1,07 & 1,003 \\
\hline 2011 & 43808,5 & 1,02 & 2011 & 1,28 & 1,21 & 1,02 \\
\hline 2012 & 43355,81 & 0,99 & 2012 & 1,28 & 0,99 & 1,04 \\
\hline 2013 & 43522,35 & 1,003 & 2013 & 1,12 & 1,145 & 1,03 \\
\hline 2014 & 44213,88 & 1,016 & 2014 & 1,38 & 1,03 & 1,03 \\
\hline 2015 & 45698,5 & 1,03 & 2015 & 1,08 & 0,94 & 1,08 \\
\hline 2016 & 46339,12 & 1,014 & & & & \\
\hline 2017 & 46681,17 & 1,007 & & & & \\
\hline 2018 & 47193,58 & 1,01 & & & & \\
\hline
\end{tabular}

Таблица 5

\section{Исходная информация по Германии - ВВП на одного работающего (в долларах)}

\begin{tabular}{|c|c|c|c|c|c|c|}
\hline Год & $\begin{array}{c}\text { Значения } Y_{i} \text { ВВП } \\
\text { ндного работающего } \\
\text { (в долларах 2011 г.) }\end{array}$ & $\begin{array}{c}\text { Значения } \\
Y_{i-1} Y_{i-1}\end{array}$ & $\begin{array}{c}\text { Модель 1. } \\
\text { Значения } \\
X 1\end{array}$ & $\begin{array}{c}\text { Модель 2. } \\
\text { Значения } \\
X 1\end{array}$ & $\begin{array}{c}\text { Модель 3. } \\
\text { Значения } \\
X 1\end{array}$ & $\begin{array}{c}\text { Модель 4. } \\
\text { Значения } \\
X 1\end{array}$ \\
\hline 2005 & 85661,28 & & & & & \\
\hline 2006 & 87229,72 & 1,018 & 1,012 & 1,264 & 1,533 & 1,128 \\
\hline 2007 & 88006,34 & 1,009 & 1,044 & 1,213 & 1,531 & 1,084 \\
\hline 2008 & 87918,29 & 0,999 & 1,058 & 1,152 & 1,740 & 1,038 \\
\hline 2009 & 83010,62 & 0,944 & 1,288 & 0,918 & 1,382 & 0,882 \\
\hline 2010 & 85500,64 & 1,030 & 1,206 & 0,982 & 1,276 & 1,011 \\
\hline 2011 & 86557,4 & 1,012 & 1,130 & 1,031 & 1,225 & 1,067 \\
\hline 2012 & 86373,15 & 0,998 & 0,998 & 1,216 & 1,367 & 1,198 \\
\hline 2013 & 85870,05 & 0,994 & 1,125 & 1,074 & 1,307 & 1,037 \\
\hline 2014 & 86898,33 & 1,012 & 1,059 & 1,018 & 1,231 & 1,015 \\
\hline 2015 & 87941,91 & 1,012 & 0,983 & 1,019 & 1,162 & 1,049 \\
\hline 2016 & 88612,2 & 1,008 & & & & \\
\hline 2017 & 89748,2 & 1,013 & & & & \\
\hline 2018 & 91357,92 & 1,018 & & & & \\
\hline
\end{tabular}


1.3. Функция отклика $\mathbf{Y 3}$ - ВВП на одного работающего (в долларах).

*Германия. Исходная информация представлена в таблице 5.

1) Модель 1. X1 - приращение объема экспорта продуктов питания (за три года, в \% от объема экспорта). Статистические характеристики качества модели:

$$
\begin{gathered}
b 1=0,91 ; \sigma_{\mathrm{b} 1}=0,027 ; b 1 / \sigma_{\mathrm{b} 1}=33,74 ; \\
R^{2}=0,99 ; R_{\text {ск }}^{2}=0,88 ; F \text { кр }=1138,2 . \\
\text { Значимость } F \text { кр }=6,51 \mathrm{E}-10 .
\end{gathered}
$$

2) Модель 2. X1 - приращение объема экспорта товаров и услуг (за три года, в \% от объема экспорта). Статистические характеристики качества модели:

$$
\begin{gathered}
b 1=0,91 ; \sigma_{\mathrm{b1}}=0,03 ; b 1 / \sigma_{\mathrm{b} 1}=27,8 ; \\
R^{2}=0,99 ; R_{\text {ск }}^{2}=0,88 ; F \text { кр }=770,1 . \\
\text { Значимость } F \text { кр }=3,07 \mathrm{E}-09 .
\end{gathered}
$$

3) Модель 3. X1 - приращение объема экспорта услуг ИКТ (за три года, в \% от экспорта услуг). Статистические характеристики качества модели:

$$
\begin{gathered}
b 1=0,72 ; \sigma_{\mathrm{b} 1}=0,03 ; b 1 / \sigma_{\mathrm{b} 1}=23,2 ; \\
R^{2}=0,98 ; R_{\text {ск }}^{2}=0,87 ; F \mathrm{\kappa p}=539,9 . \\
\text { Значимость } F \text { кр }=1,25 \mathrm{E}-08 .
\end{gathered}
$$

4) Модель 4. X1 - Промышленность и строительство: приращение добавленной стоимости на одного работника (за три года). Статистические характеристики качества модели:

$$
\begin{gathered}
b 1=0,95 ; \sigma_{\mathrm{b} 1}=0,025 ; b 1 / 6_{\mathrm{b} 1}=37,8 ; \\
R^{2}=0,99 ; R_{\text {ск }}^{2}=0,88 ; F \text { кр }=1432,1 . \\
\text { Значимость } F \text { кр }=2,61 \mathrm{E}-10 .
\end{gathered}
$$

5) Модель 5. X1 - приращение объема экспорта товаров и услуг (за три года, в \% от объема экспорта); $\boldsymbol{X 2}$ - приращение объема экспорта продуктов питания (за три года, в \% от объема экспорта). Статистические характеристики качества модели:

$$
\begin{gathered}
b 1=0,4 ; b 2=0,52 ; \sigma_{\mathrm{b} 1}=0,09 ; \sigma_{\mathrm{b} 2}=0,09 ; \\
b 1 / \sigma_{\mathrm{b} 1}=4,4 ; b 2 / \sigma_{\mathrm{b} 2}=5,7 ; R^{2}=0,99 ; \\
R_{\mathrm{ck}}^{2}=0,87 ; \text { Fर }=1748,9
\end{gathered}
$$

Значимость Гкр $=3,56 \mathrm{E}-10$.

*Россия. Исходная информация представлена в таблице 6.

1) Модель 1. X1 - приращение доли технического персонала в НИОКР (за один год, на миллион человек). Статистические характеристики качества модели:

$$
\begin{aligned}
& b 1=1,027 ; \sigma_{b 1}=0,015 ; b 1 / \sigma_{b 1}=68,87 \text {; } \\
& R^{2}=0,99 ; R^{2}=0,9 ; F_{\text {кр }}=4743,2 \text {. } \\
& \text { Значимость } F \text { кр }=1,02 E-14 \text {. }
\end{aligned}
$$

2) Модель 2. $X 1$ - приращение количества занятых в промышленности (за два года, в \% от общей занятости). Статистические характеристики качества модели:

$$
\begin{gathered}
b 1=1,04 ; \sigma_{b 1}=0,012 ; b 1 / \sigma_{b 1}=85,87 ; \\
R^{2}=0,99 ; R_{\text {ск }}^{2}=0,9 ; F \text { кр }=7372,8 . \\
\text { Значимость } F \text { кр }=1,12 \mathrm{E}-15 .
\end{gathered}
$$

3) Модель 3. $X 1$ - приращение объема торговли (за один год, в \% от ВВП). Статистические характеристики качества модели:

$$
\begin{gathered}
b 1=1,03 ; \sigma_{\mathrm{b1}}=0,015 ; b 1 / \sigma_{\mathrm{b} 1}=68,5 ; \\
R^{2}=0,99 ; R_{\text {ск }}^{2}=0,9 ; F \text { кр }=4697,2 . \\
\text { Значимость } F \text { кр }=1,07 \mathrm{E}-14 .
\end{gathered}
$$

*Швеция. Исходная информация представлена в таблице 7.

1) Модель 1. X1 - приращение доли промышленности в сформированном ВВП (за три года, в \% от ВВП). Статистические характеристики качества модели:

$$
\begin{gathered}
b 1=1,053 ; \sigma_{\mathrm{b1}}=0,018 ; b 1 / \sigma_{\mathrm{b1}}=56,75 ; \\
R^{2}=0,99 ; R_{\text {ск }}^{2}=0,89 ; F_{\mathrm{Kp}}=3221,1 \\
\text { Значимост } F \text { кр }=1,03 \mathrm{E}-11 .
\end{gathered}
$$

2) Модель 2. $X 1$ - Услуги: приращение добавленной стоимости (за три года, в $\%$ от ВВП0). Статистические характеристики качества модели:

$$
\begin{gathered}
b 1=0,99 ; \sigma_{b 1}=0,01 ; b 1 / \sigma_{b 1}=95,8 ; \\
R^{2}=0,99 ; R^{2}=0,89 ; F \kappa p=9181,3 . \\
\text { Значимость } F \text { кр }=1,57 E-13 .
\end{gathered}
$$

3) Модель 3. X1 - Услуги: приращение добавленной стоимости на одного работника (за три года, в ценах 2010 г.). Статистические характеристики качества модели:

$$
\begin{gathered}
b 1=0,98 ; \sigma_{b 1}=0,01 ; b 1 / \sigma_{\mathrm{b1}}=88,1 ; \\
R^{2}=0,99 ; R_{\text {ск }}^{2}=0,89 ; F \text { кр }=7758,03 . \\
\text { Значимость } F \text { кр }=3,08 \mathrm{E}-13 .
\end{gathered}
$$

4) Модель 4. X1 - Промышленность: приращение добавленной стоимости на одного работника (за три года, в ценах 2010 г.). Статистические характеристики качества модели:

$$
\begin{gathered}
b 1=0,96 ; \sigma_{b 1}=0,027 ; b 1 / \sigma_{\mathrm{b} 1}=35,76 ; \\
R^{2}=0,99 ; R_{\text {ск }}^{2}=0,88 ; F \kappa p=1278,8 . \\
\text { Значимость } F \text { кр }=4,1 \mathrm{E}-10 .
\end{gathered}
$$

5) Модель 5. X1 - приращение доли промышленности в сформированном ВВП (за три года, в \% от ВВП); $\boldsymbol{X} 2-$ Услуги: приращение добавленной стоимости (за три года, в \% от ВВП0). Статистические характеристики качества модели:

$$
b 1=0,33 ; b 2=0,68 ; \sigma_{b 1}=0,12 ; \sigma_{b 2}=0,11 \text {; }
$$$$
b 1 / \sigma_{\mathrm{b} 1}=2,8 ; b 2 / \sigma_{\mathrm{b} 2}=6,1 ; R^{2}=0,99 \text {; }
$$ 
Исходная информация по России - ВВП на одного работающего (в долларах)

\begin{tabular}{|c|c|c|c|c|c|}
\hline Год & $\begin{array}{c}\text { Значения } Y_{i} \text { ВВП } \\
\text { наного работающего } \\
\text { (в долларах 2011 г.) }\end{array}$ & $\begin{array}{c}\text { Значения } \\
Y_{i} Y_{i-1}\end{array}$ & $\begin{array}{c}\text { Модель 1. } \\
\text { Значения } X 1\end{array}$ & $\begin{array}{c}\text { Модель 2. } \\
\text { Значения } X 1\end{array}$ & $\begin{array}{c}\text { Модель 3. } \\
\text { Значения } X 1\end{array}$ \\
\hline 2003 & 36341,8 & & & & 0,981 \\
\hline 2004 & 38378,18 & 1,056 & 0,991 & 0,979 & 1,002 \\
\hline 2005 & 40208,28 & 1,047 & 0,936 & 0,967 & 0,965 \\
\hline 2006 & 43237,68 & 1,075 & 1,002 & 0,984 & 0,944 \\
\hline 2007 & 45751,89 & 1,058 & 0,987 & 0,98 & 1,032 \\
\hline 2008 & 47913,42 & 1,047 & 0,952 & 0,938 & 0,907 \\
\hline 2009 & 45156,25 & 0,942 & 0,974 & 0,951 & 1,039 \\
\hline 2010 & 46807,55 & 1,036 & 1,001 & 0,947 & 0,951 \\
\hline 2011 & 48744,3 & 1,041 & 1,035 & 1,011 & 0,983 \\
\hline 2012 & 50108,36 & 1,027 & 0,969 & 0,999 & 0,98 \\
\hline 2013 & 51323,67 & 1,024 & 1,018 & 1,002 & 1,034 \\
\hline 2014 & 51726,18 & 1,007 & 1,028 & 0,978 & 1,032 \\
\hline 2015 & 50663,28 & 0,979 & 0,975 & & \\
\hline 2016 & 50668,7 & 1,000 & & & \\
\hline 2017 & 51812,7 & 1,022 & & & \\
\hline 2018 & 53011,71 & 1,023 & & & \\
\hline
\end{tabular}

Таблица 7

Исходная информация по Швеции - ВВП на одного работающего (в долларах 2011 г.)

\begin{tabular}{|c|c|c|c|c|c|c|}
\hline Год & $\begin{array}{c}\text { Значения } Y_{i} \text { ВВП } \\
\text { ндного работающего } \\
\text { (в долларах 2011 г.) }\end{array}$ & $\begin{array}{c}\text { 3начения } \\
Y_{i-1}\end{array}$ & $\begin{array}{c}\text { Модель 1. } \\
\text { Значения } \\
X 1\end{array}$ & $\begin{array}{c}\text { Модель 2. } \\
\text { Значения } \\
X 1\end{array}$ & $\begin{array}{c}\text { Модель 3. } \\
\text { Значения } \\
X 1\end{array}$ & $\begin{array}{c}\text { Модель 4. } \\
\text { Значения } \\
X 1\end{array}$ \\
\hline 2005 & 85737,61 & & & & & \\
\hline 2006 & 88128,66 & 1,028 & 1,030 & 0,993 & 1,067 & 1,209 \\
\hline 2007 & 88861,47 & 1,008 & 1,020 & 0,993 & 1,028 & 1,134 \\
\hline 2008 & 87200,57 & 0,981 & 0,989 & 1,003 & 1,010 & 1,052 \\
\hline 2009 & 84334,46 & 0,967 & 0,890 & 1,042 & 0,979 & 0,913 \\
\hline 2010 & 89010,56 & 1,055 & 0,951 & 1,017 & 1,003 & 1,026 \\
\hline 2011 & 89535,91 & 1,006 & 0,963 & 1,012 & 1,020 & 1,069 \\
\hline 2012 & 88769,13 & 0,991 & 1,005 & 1,006 & 1,041 & 1,128 \\
\hline 2013 & 88870,56 & 1,001 & 0,907 & 1,050 & 1,027 & 0,962 \\
\hline 2014 & 90521,23 & 1,019 & 0,916 & 1,045 & 1,032 & 0,984 \\
\hline 2015 & 93596,8 & 1,034 & 0,914 & 1,038 & 1,075 & 0,998 \\
\hline 2016 & 95488,16 & 1,020 & & & & \\
\hline 2017 & 96448,58 & 1,010 & & & & \\
\hline 2018 & 98264,89 & 1,019 & & & & \\
\hline
\end{tabular}


$R^{2}=0,87 ;$ Гкр $=8179,7$.

Значимость Fкр = 1,62E-12.

6) Модель 6. X1 - Услуги: приращение добавленной стоимости (за три года, в $\%$ от ВВП0); $\boldsymbol{X 2}$ - Промышленность: приращение добавленной стоимости на одного работника (за три года, в ценах 2010 г.) Статистические характеристики качества модели:

$b 1=0,796 ; b 2=0,19 ; \sigma_{\mathrm{b} 1}=0,085 ; \sigma_{\mathrm{b} 2}=0,08 ;$

$b 1 / 6_{\mathrm{b} 1}=9,34 ; b 2 / 6_{\mathrm{b} 2}=2,3 ; R^{2}=0,99$; $R^{2}{ }_{\mathrm{ck}}=0,87 ;$ Fкр $=6813,1$.

Значимость Fкр $=3,07-12$.

Выводы. В процессе построения моделей для прогнозирования динамики индикаторов уровня развития экономики страны виервыlе:

1) установлено определяющее, статистически значимое и содержательно обоснованное влияние на функции отклика выделенных групп факторов - независимых переменных (в большинстве случаев у $\boldsymbol{b}$-коэффициентов отношение $\boldsymbol{b} / \mathbf{6}_{b i}>>2$ );

2) построены регрессионные модели для прогнозирования динамики индикаторов уровня развития экономики страны *лучшего качества (при оценке по стандартным критериям статистической значимости $-\boldsymbol{R}_{\text {скорр }}^{2} \approx \mathbf{0 , 9}$; Fкр >> 100) и *с использованием открытых официальных статистических данных;

3) обеспечена возможность, базируясь на сформированном в процессе исследований подмножестве значимых факторов, оценивать потребительское качество и прогнозные свойства созданных моделей, проводить расчеты с целью минимизации затрат ресурсов на оптимальный выбор и реализацию проектов, направленных на повышение значений показателей-функций отклика Ү1-Y3, характеризующих уровень развития экономики страны.

2. Содержательное обоснование состава определяющих факторов. Обоснованность включения в состав независимых переменных при построении регрессионных моделей всех выявленных в процессе исследований факторов подтверждена статистически. Содержательная обоснованность также не подлежит сомнению. Покажем это на нескольких примерах.

Функция отклика - Y1 Ожидаемая продолжительность жизни (ОПЖ) граждан в Швеции $\geq 80$ лет. И это в стране, население которой трудно заподозрить в том, что все граждане в обязательном порядке придерживаются средиземноморской диеты или питаются преимущественно морскими водорослями, как часть японских островных долгожителей. Тогда в чем же состоит скандинавский секрет долгожительства?

Нам представляется, что состав независимых переменных дает не только статистически, но и содержательно обоснованный ответ на этот вопрос. Действительно, ведь очевидно, что чем больше государство инвестирует в здравоохранение (модели 1 и 2), тем больше вероятность того, что в стране будут более квалифицированные медицинские работники, более качественная медицинская аппаратура и оптимальная организация медицинского обслуживания населения, да и модель 3 выглядит не менее обоснованной. Разве прирост оплаты труда работников не увеличивает возможность и вероятность покупки более эффективных, но дорогостоящих лекарств (и даже увеличивает вероятность внутреннего, душевного успокоения просто из-за наличия такой возможности) и т.д.?

Что касается участия государства в финансировании здравоохранения, то практически аналогичные результаты относительно функции отклика $\boldsymbol{Y 1}$ получены нами ранее при проведении исследований на примере Германии и России [14].

Если теперь обратиться к составу независимых переменных в моделях для прогнозирования функций $\boldsymbol{Y 2}$ (ВВП на душу населения, ППС; в постоянных международных долларах 2011 г.) и $Y 3$ (ВВП на одного работающего, в долларах 2011 г.), то и здесь обнаружим полное совпадение результатов статистического и содержательного анализов. Так, по Германии в составе независимых переменных в моделях для прогнозирования $\boldsymbol{Y 2}$ и $\boldsymbol{Y 3}$ оказались факторы-показатели: «относительное приращение валового накопления основного капитала», «приращение объема экспорта товаров и услуг», «приращение объема экспорта продуктов питания», «приращение объема экспорта услуг ИКТ», «промышленность и строительство: приращение добавленной стоимости на одного работника» и другие факторы аналогичного экономического содержания. 
Спрашивается: разве можно, не нарушая базовых постулатов экономической теории, исключить любой из перечисленных показателей как недостаточно содержательно обоснованный?

Вывод. Содержательная обоснованность включения в состав независимых переменных группы перечисленных определяющих показателей (для прогнозирования динамики индикаторов уровня развития экономики) не вызывает сомнения.

3. Оценка прогнозных свойств построенных регрессионных моделей. Результаты расчета характеристик потребительского качества построенных моделей рассмотрим на примере Швеции (функция Y3 - ВВП на одного работающего, в долларах 2011 г. (см. таблицу 7)).

Объективная сравнительная оченка прогнозных свойств построенных моделей представлена в таблице 8.

Если ограничить интервал прогнозирования 2016, 2017 и 2018 годами, то результаты оценки прогнозных свойств моделей с выделенным составом независимых переменных и с лагом 3 года окажутся, как видно из представленных в таблице 9 данных, еще более успешными.

Аналогично, используя построенные модели и исходные данные, представленные в таблицах 1-6, можно оценить потребительское качество построенных прогнозных моделей для различных стран, включая Германию и Россию, и исследуемых функций.

Выводы. Использование в составе независимых переменных выделенной группы определяющих факторов позволило:

1) получить среднюю ошибку прогноза на 2016-2018 годы по 6-ти моделям в пределах от $7 \%$ до $0,3 \%$, в том числе по 2-м моделям - менее одного процента, а за весь период с 2009 по 2018 годы — от 6 до 1,5\%;

2) подтвердить прикладную полезность применения *совокупности построенных моделей и *метода выделения искомого подмножества объектов из множества большой мощности $[8,13]$;

3) обеспечить органам государственной власти всех уровней уникальные возможности для совершенствования управления экономическим развитием страны, ориен- тируясь на созданные конкретные модели и конкретные независимые переменные, в том числе используя модели и показателифакторы при реализации гибкой налоговой и таможенной политики, информировании субъектов рынка о динамике иен на товары и услуги на внутренних и внешних рынках, оказании услуг юридическим лицам при поиске товаров и технологических прочессов с минимальной ресурсоемкостью и др. $[5,6$, $9,12,15,16]$.

\section{4. Имитационное моделирование для} оценки ошибки функций отклика. Как показано в [10], основное влияние на величину ошибки функции отклика при построении регрессионных моделей для описания и/или прогнозирования процессов оказывают структура регрессионной модели, состав и точность измерения или фиксации независимых переменных.

Описанный метод позволяет оценивать статистические характеристики распределения функции отклика (математическое ожидание, коэффициент вариации, среднеквадратическое отклонение, асимметрию, эксцесс, характер распределения в виде гистограммы и таблицы и др.) при различных значениях ошибок независимых переменных и разной структуре уравнения регрессии.

Зная величину ошибок измерения (фиксации) независимых переменных $\boldsymbol{X}_{\boldsymbol{i}}$, значения $\boldsymbol{b}$-коэффициентов и $\boldsymbol{\sigma}_{\boldsymbol{b} \boldsymbol{i}}$ и выполнив имитационное моделирование, легко оценить ошибку функции отклика $\boldsymbol{Y}$.

Здесь на примере моделей для прогнозирования динамики функции «ВВП на одного работающего (в долларах)» на данных по Швеции выполним оценку ошибок функций отклика по построенным моделям.

Для реализации моделирования необходимая исходная информация по $\boldsymbol{b}$-коэффициентам получена в процессе построения регрессионных моделей (см. Статистические характеристики качества). Что касается независимых переменных $\boldsymbol{X}_{\boldsymbol{i}}$, то, поскольку в базе данных Всемирного банка отсутствуют сведения о стандартных ошибках социально-экономических показателей развития стран мира, $\boldsymbol{\sigma}_{\boldsymbol{x}}$ примем равными сначала 0,01 (1\%), а затем 0,03 (3\%). В таблице 10 представлены полученные в результате ими- 


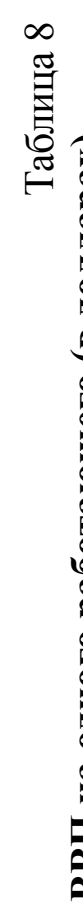

舀

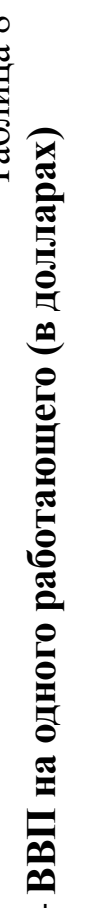

\section{8}

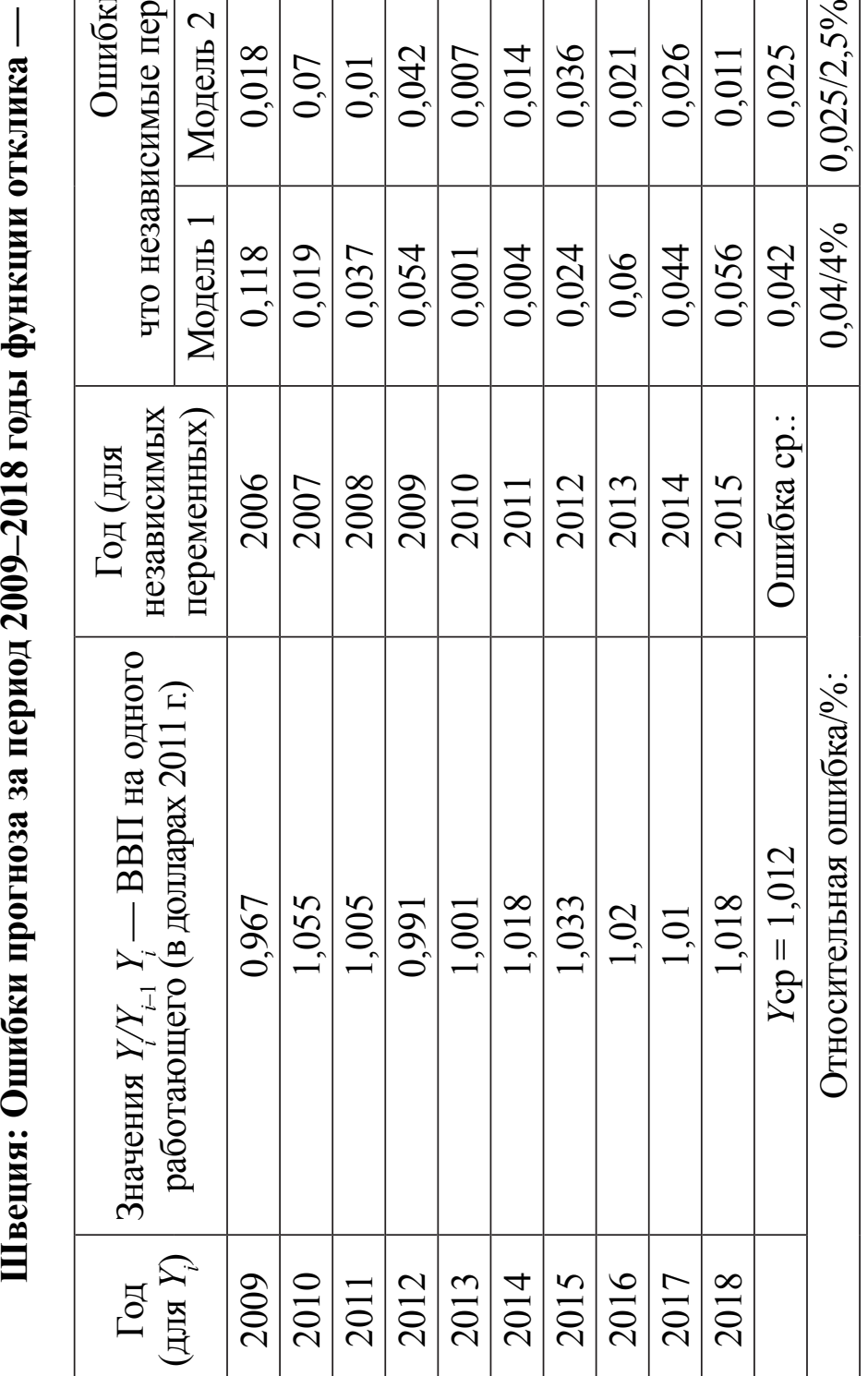

藏

至

约

药

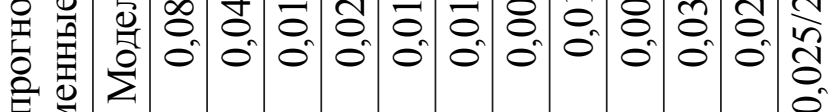

0

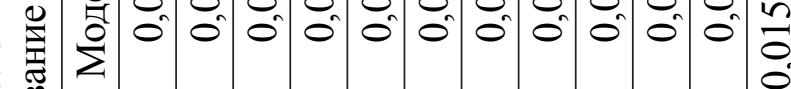

要
急

政

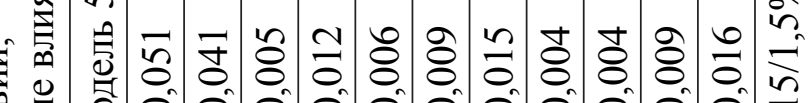

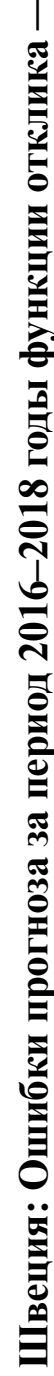

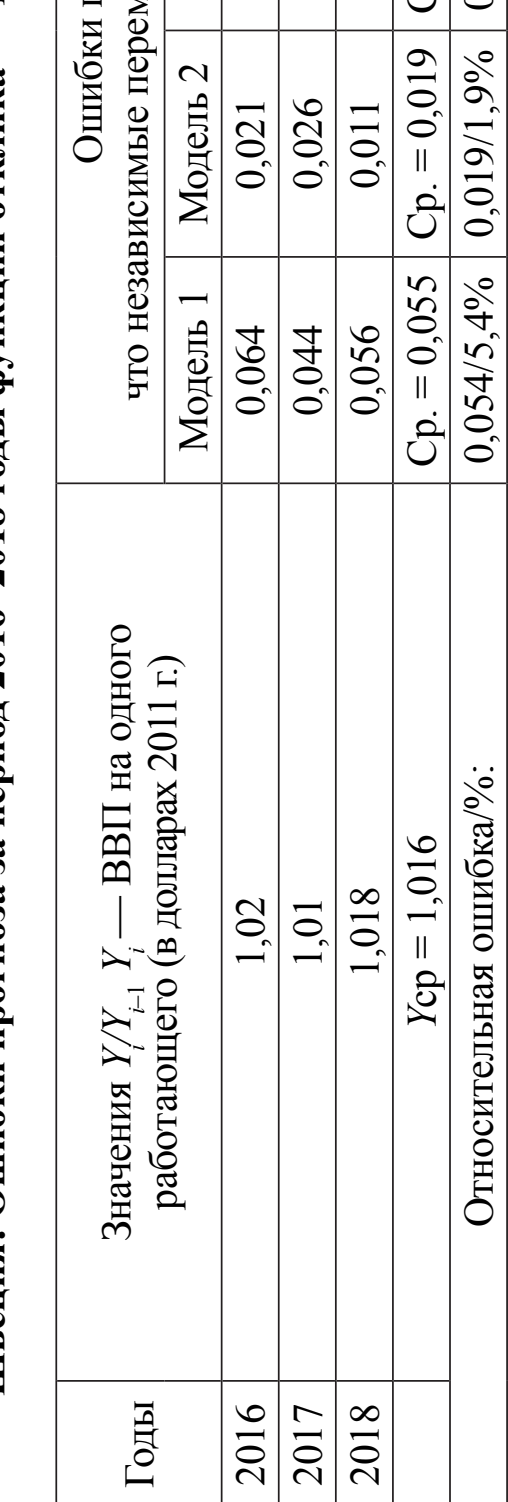


тационного моделирования характеристики функции отклика $Y 3$ (Швеция).

Предположим теперь, что нас интересует ошибка функции отклика для модели 6, включающей две независимые переменные $x 1$ и $x 2$. Причем, $\boldsymbol{6}_{x 2}$ примем равным не 0,01, a 0,03 . Тогда значение ошибки функции отклика из-за изменения структуры уравнения и увеличения ошибки $x 2$ возрастет весьма существенно (см. табл. 11).

В таблице 12 показано распределение значений $\mathbf{y 6}$ (модель 6) в результате имитационного моделирования.

Выводы. В результате применения имитационного моделирования для оценки ошибки функции отклика:
1) обнаружено, что величина ошибок функиий отклика даже у моделей с близкими численными значениями статистических характеристик качества (отношение $b_{i} / \sigma_{b i}$, $R_{\text {скорр }}^{2}, F$ кр) существенно отличается (см. численные значения статистических характеристик и ошибок у моделей 1 и 2);

2) установлено, что снижение достоверности измерения (фиксации) численных значений только одной из независимых переменных $(X 2)$ у модели с лучшими статистическими характеристиками качества (модель 6) приводит к значительному росту ошибки функции отклика;

3) подтверждено весьма существенное влияние и структуры регрессионной модели,

Результаты моделирования у1 (модель 1) и у2 (модель 2);

Таблица 10 функция отклика Y3 - ВВП на одного работающего (в долларах) по данным Швеции

\begin{tabular}{|l|c|c|}
\hline \multicolumn{1}{|c|}{ Параметр } & \multicolumn{2}{c|}{ Значение } \\
\hline Переменная & $y 1$ & $y 2$ \\
\hline Число итераций & 1000 & 1000 \\
\hline Среднее & 1,011 & 1,012 \\
\hline Среднеквадратическое отклонение & 0,021 & 0,014 \\
\hline Асимметрия & 0,045 & 0,019 \\
\hline Эксцесс & $-0,010$ & $-0,154$ \\
\hline Минимум & 0,947 & 0,967 \\
\hline Максимум & 1,079 & 1,059 \\
\hline Модальный интервал & $1,007: 1,019$ & $1,008: 1,017$ \\
\hline
\end{tabular}

Таблица 11

Характеристики распределения значений Y6 (функция отклика модели 6), полученные в результате имитационного моделирования

\begin{tabular}{|l|c|}
\hline \multicolumn{1}{|c|}{ Параметр } & Значение \\
\hline Переменная & $y 6$ \\
\hline Комментарий & 1000 \\
\hline Число итераций & 1,010 \\
\hline Среднее & 0,123 \\
\hline Среднеквадратическое отклонение & 0,072 \\
\hline Асимметрия & $-0,057$ \\
\hline Эксцесс & 0,626 \\
\hline Минимум & 1,410 \\
\hline Максимум & $0,983: 1,054$ \\
\hline Модальный интервал & 1,005 \\
\hline Медиана & \\
\hline
\end{tabular}


и разницы в величине ошибок независимых nеременных на величину ошибки функции отклика.

Заключение. В результате проведенных исследований впервые:

1) доказано определяющее, статистичес$к и$ значимое и содержсательно обоснованное влияние на динамику индикаторов уровня развития страны выделенных групп факторов- независимых переменных (в большинстве случаев у $\boldsymbol{b}$-коэффициентов отношение $\boldsymbol{b}_{\boldsymbol{i}} / \mathbf{6}_{\boldsymbol{b i}}>\mathbf{2}$ ); построены регрессионные модели для прогнозирования динамики ВВП страны и ожсидамой продолюсительности жизни граждан *лучшего качества (при оценке по стандартным критериям статистической значимости $-\boldsymbol{R}_{\text {скорр }}^{2} \approx \mathbf{0 , 9 ;}$ Fкр $>>100$ );

2) установлено, что использование в моделях оригинального состава независимых переменных позволило: а) получить среднюю ошибку за 10-летний период прогноза по исследуемым странам менее $5 \%$ при лаге независимых переменных от одного до трех лет; б) подтвердить прикладную полезность применения *совокупности построенных моделей и *метода выделения искомого подмножества объектов из множества большой мощности; в) обеспечить органам государственной власти возможности для совершенствования управления экономическим развитием страны и административно-территориальных образований;
3) обнаружено, что величина ошибок функичий отклика даже у моделей с близкими численными значениями статистических характеристик качества существенно отличается. Одновременно установлено, что снижение достоверности измерения (фиксации) численных значений только одной из независимых переменных у модели с лучшими статистическими характеристиками качества приводит к значительному росту ошибки функции отклика;

4) определено, что полученное в процессе исследований доказательство статистической и содержательной обоснованности влияния на динамику индикаторов уровня развития экономики выделенного оригинального подмножества определяющих факторов обеспечило возможность «властным структурам, обладаюшим суверенными полномочиями, решать вопросы организачии общества в масштабах страньл) (Универсальный энциклопедический словарь. - М.: БРЭ, 2002. - 1551 с.), действующим в различных странах мира, более целенаправленно и более активно содействовать ускоренному развитию экономики, ориентируясь на созданные конкретные модели и конкретные независимые переменные, в том числе используя такие модели: при реализащии гибкой налоговой и таможенной политики, при информировании субъектов рынка о динамике иен на товары и услуги на внутренних и внешних рынках, при оказании услуг юридчческим ли-

Распределение значений у6 (модель 6)

\begin{tabular}{|c|c|c|c|c|}
\hline$X_{\min }$ & $X_{\max }$ & Частота & Вероятность & Накопленная \\
\hline 0,626 & 0,698 & 6 & 0,006 & 0,006 \\
\hline 0,698 & 0,769 & 17 & 0,017 & 0,023 \\
\hline 0,769 & 0,840 & 55 & 0,055 & 0,078 \\
\hline 0,840 & 0,911 & 138 & 0,138 & 0,216 \\
\hline 0,911 & 0,983 & 206 & 0,206 & 0,422 \\
\hline 0,983 & 1,054 & 213 & 0,213 & 0,635 \\
\hline 1,054 & 1,125 & 194 & 0,194 & 0,829 \\
\hline 1,125 & 1,196 & 99 & 0,099 & 0,928 \\
\hline 1,196 & 1,268 & 51 & 0,051 & 0,979 \\
\hline 1,268 & 1,339 & 17 & 0,017 & 0,996 \\
\hline 1,339 & 1,410 & 4 & 0,004 & 1,000 \\
\hline
\end{tabular}


цамм в поиске товаров и технологических произессов с минимальной ресурсоемкостью и др.

\section{Литература}

1. Хубаев Г.Н., Щербакова К.Н., Сидоренко Д. С. Веб-приложение для сравнительной оценки, анализа динамики и прогнозирования уровня жизни населения субъектов РФ // Свидетельство о государственной регистрации программы для ЭВМ №2019619362 [Электронный ресурс] - М.: Роспатент, 2019. — Режим доступа: http://uroven-zhizni.ru.

2. Хубаев Г.Н., Гулаков С.В. Информационная система для сравнительной оценки качества жизни населения субъектов РФ «Рейтинг субъектов РФ» // Свидетельство об официальной регистрации программы для ЭВМ №2002384636. — М.: РОСПАТЕНТ, 2006.

3. Хубаев Г.Н. Экспресс-оценка качества жизни населения муниципальных образований // Обозрение прикладной и промышленной математики. - М.: Редакция журнала «ОП и ПМ», 2006. - Т. 13. - Вып. 3. C. 556-560.

4. Хубаев Г.Н. Социально-экономическая обстановка в России и приоритетные направления экономической политики // Экономико-организационные проблемы проектирования и применения информационных систем: Материалы 3-й Межгосударственной научнопрактической конференции (Ростов-на-Дону, 25-26 ноября 1998 г.). — Ростов-на-Дону: РГЭА, 1998. - С. 8-22.

5. Хубаев Г.Н. Рынок услуг: оценка статистических характеристик распределения затрат финансовых ресурсов и времени покупателей // РИСК: Ресурсы, информация, снабжение, конкуренция. — 2017. — №2. - С. 90-96.

6. Хубаев Г.Н. Оценка резервов снижения ресурсоёмкости товаров и услуг: методы и инструментальные средства // ПРИКЛАДНАЯ ИНФОРМАТИКА. - 2012. №2 (38). - С. 84-90.

7. Хубаев Г.Н. Методы формирования согласованного коллективного выбора в процессе экспертизы (на примере ранжирования способов решения сложных проблем)// Бюллетень науки и практики. - 2017. №7 (20). - C. 59-77.

8. Хубаев Г.Н. Метод выделения искомого подмножества объектов из множества большой мощности // Гуманитарные, социально-экономические и общественные науки. - 2019. - №8. - С. 206-211.

9. Хубаев Г.Н. Как уменьшить вероятность ошибки при выборе приоритетных направлений социального и экономического развития страны [Электронный ресурс]// Бюллетень науки и практики. - 2019. №12. - С. 265-280. - Режим доступа: https://doi.org/10.33619/2414-2948/49/31.

10. Хубаев Г.Н. Имитационное моделирование при выборе состава факторов и структуры уравнения регрессии // ПРИКЛАДНАЯ ИНФОРМАТИКА. — 2010. — №6. - С. 83-89.

11. Хубаев Г.Н. Вид пропасти заставляет строить мост // Экономика и жизнь. - Юг. 1999. - №9-12.

12. Хубаев Г.Н. Государство и рынок: как государство может усилить позитивное взаимовлияние субъектов рынка [Электронный pecypc] // Бюллетень науки и практики. 2020. — №6. - С. 175-198. — Режим доступа: https://doi.org/10.33619/2414-2948/55/22.

13. Khubaev G.N. Method of isolating a desired subset of objects from a set of greater power [Electronic resource] // Scientific research of the SCO countries: synergy and integration (Beijing, China 31 July 2019). - Beijing, 2019. P. 50-57. - URL: https://doi.org/10.34660/ INF.2019.13.33962.

14. Khubaev G.N. Models for forecasting the expected life of the population of the RUSSIAN FEDERATION and GERMANY [Electronic resource] // International Conference «Process Management and Scientific Developments» (Birmingham, United Kingdom, December 19, 2019). Part 1. - Birmingham, UK, 2019. - P. 54-60. — URL: https://doi. org/10.34660/INF.2019.3.44512.

15. Khubaev G.N. How to reduce the probability of errors when selecting the priority directions of the social and economic development of the country [Electronic resource] // Materials of the International Conference «Process Management and Scientific Developments» (Birmingham, United Kingdom, November 14, 2019). Part 3. - Birmingham, UK, 2019. P. 9-19. - URL: https://doi.org/10.34660/ INF.2019.1.40958.

16. Khubaev G. N. How the state can strengthen the positive interaction of market participants: methods and tools [Electronic resource] // Mate- 
rials of the International Conference «Scientific research of the SCO countries: synergy and integration» (May 14, 2020. Beijing, PRC). - Beijing, China, 2020. - P. 30-38. - URL: https:// doi.org/10.34660/INF.2020.28.64076.

\section{References}

1.Hubaev G. N., Shherbakova K. N.,Sidorenko D.S. Veb-prilozhenie dlja sravnitel'noj ocenki, analiza dinamiki i prognozirovanija urovnja zhizni naselenija subektov RF [Web application for comparative assessment, analysis of dynamics and forecasting of the standard of living of the population of the subjects of the Russian Federation] // Svidetel'stvo o gosudarstvennoj registracii programmy dlja JeVM №2019619362 [Certificate of state registration of a computer program №2019619362] [Jelektronnyj resurs] - Moscow: Rospatent, 2019. — URL: http://uroven-zhizni.ru.

2. Hubaev G.N., Gulakov S. V. Informacionnaja sistema dlja sravnitel'noj ocenki kachestva zhizni naselenija subektov RF «Rejting subektov RF» [Information system for comparative assessment of the quality of life of the population of the subjects of the Russian Federation «Rating of subjects of the Russian Federation»] // Svidetel'stvo ob oficial'noj registracii programmy dlja JeVM №2002384636 [Certificate of official registration of the computer program №2002384636]. — Moscow: ROSPATENT, 2006.

3. Hubaev G. N. Jekspress-ocenka kachestva zhizni naselenija municipal'nyh obrazovanij [Express assessment of the quality of life of the population of municipalities] // Obozrenie prikladnoj i promyshlennoj matematiki [Review of applied and industrial mathematics]. Moscow: Redakcija zhurnala «OP i $\mathrm{PM} »$, 2006. - Vol. 13. - Issue 3. - Pp. 556-560.

4. Hubaev G. N. Social'no-jekonomicheskaja obstanovka v Rossii i prioritetnye napravlenija jekonomicheskoj politiki [Socio-economic situation in Russia and priority directions of economic policy] // Jekonomiko-organizacionnye problemy proektirovanija i primenenija informacionnyh sistem: Materialy 3-j Mezhgosudarstvennoj nauchno-prakticheskoj konferencii (Rostov-na-Donu, 25-26 nojabrja 1998 g.) [Economic and organizational problems of designing and applying information systems:
Proceedings of the 3rd Interstate scientific and practical conference (Rostov-on-Don, November 25-26, 1998)]. — Rostov-on-Don: RGJeA, 1998. - Pp. 8-22.

5. Hubaev G.N. Rynok uslug: ocenka statisticheskih harakteristik raspredelenija zatrat finansovyh resursov i vremeni pokupatelej [Service Market: evaluation of statistical characteristics of the distribution of costs of financial resources and time of buyers] // RISK: Resursy, informacija, snabzhenie, konkurencija [RISK: Resources, information, supply, competition]. - 2017. — №2. - Pp. 90-96.

6. Hubaev G. N. Ocenka rezervov snizhenija resursojomkosti tovarov i uslug: metody i instrumental'nye sredstva [The estimation of reserves of reduction of resource intensity of products and services: methods and tools] // PRIKLADNAJa INFORMATIKA [APPLIED INFORMATICS]. — 2012. — №2 (38). Pp. 84-90.

7. Hubaev G.N. Metody formirovanija soglasovannogo kollektivnogo vybora $\mathrm{v}$ processe jekspertizy (na primere ranzhirovanija sposobov reshenija slozhnyh problem) [Methods of forming a coherent collective choice in the examination process (for example the ranking solutions to complex problems)] // Bjulleten' nauki i praktiki [Bulletin of the science and practice]. — 2017. — №7 (20). — Pp. 59-77.

8. Hubaev G. N. Metod vydelenija iskomogo podmnozhestva obektov iz mnozhestva bol'shoj moshhnosti [The method of isolating the desired subset of objects of the plurality of high power]// Gumanitarnye, social'no-jekonomicheskie i obshhestvennye nauki [Humanitarian, socioeconomic and social Sciences]. — 2019. №8. - Pp. 206-211.

9. Hubaev G.N. Kak umen'shit' verojatnost' oshibki pri vybore prioritetnyh napravlenij social'nogo i jekonomicheskogo razvitija strany [How to reduce the probability of error in the selection of priority directions of social and economic development] [Jelektronnyj resurs] // Bjulleten' nauki i praktiki [Bulletin of science and practice]. — 2019. — №12. — Pp. 265-280. URL: https://doi.org/10.33619/2414-2948/49/31.

10. Hubaev G. N. Imitacionnoe modelirovanie pri vybore sostava faktorov i struktury uravnenija regressii [Imitational modeling when choosing the composition of factors and the structure of the regression equation] // 
PRIKLADNAJa INFORMATIKA [APPLIED INFORMATICS]. — 2010. — №6. - Pp. 83-89.

11. Hubaev G. N. Vid propasti zastavljaet stroit' most [The view of the abyss makes you build a bridge] // Jekonomika i zhizn' [Economy and life]. - Jug. - 1999. — №9-12.

12. Hubaev G.N. Gosudarstvo i rynok: kak gosudarstvo mozhet usilit' pozitivnoe vzaimovlijanie subektov rynka [State and market: how the state can strengthen the positive mutual influence of market subjects] [Jelektronnyj resurs] // Bjulleten' nauki i praktiki [Bulletin of science and practice]. - 2020. №6. - Pp. 175-198. - URL: https://doi. org/10.33619/2414-2948/55/22.

13. Khubaev G.N. Method of isolating a desired subset of objects from a set of greater power [Electronic resource] // Scientific research of the SCO countries: synergy and integration (Beijing, China 31 July 2019). Beijing, 2019. - P. 50-57. — URL: https://doi. org/10.34660/INF.2019.13.33962.

14. Khubaev G. N. Models for forecasting the expected life of the population of the RUSSIAN FEDERATION and GERMANY [Electronic resource] // International Conference «Process Management and Scientific Developments» (Birmingham, United Kingdom, December 19, 2019). Part 1. - Birmingham, UK, 2019. P. 54-60. - URL: https://doi.org/10.34660/ INF.2019.3.44512.

15. Khubaev G.N. How to reduce the probability of errors when selecting the priority directions of the social and economic development of the country [Electronic resource] // Materials of the International Conference «Process Management and Scientific Developments» (Birmingham, United Kingdom, November 14, 2019). Part 3. - Birmingham, UK, 2019. P. 9-19. - URL: https://doi.org/10.34660/ INF.2019.1.40958.

16. Khubaev G.N. How the state can strengthen the positive interaction of market participants: methods and tools [Electronic resource] // Materials of the International Conference «Scientific research of the SCO countries: synergy and integration» (May 14, 2020. Beijing, PRC). — Beijing, China, 2020. P. 30-38. - URL: https://doi.org/10.34660/ INF.2020.28.64076.

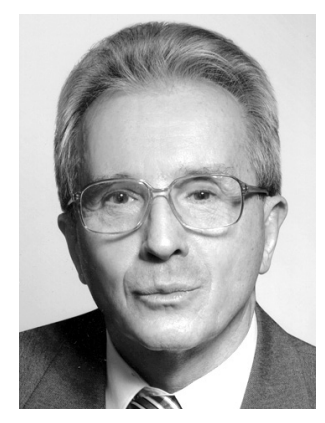

344002, г. Ростов-на-Дону, ул. Б. Садовая, 69 69 B. Sadovaya st., 344002, Rostov-on-Don, Russia E-mail: gkhubaev@mail.ru 